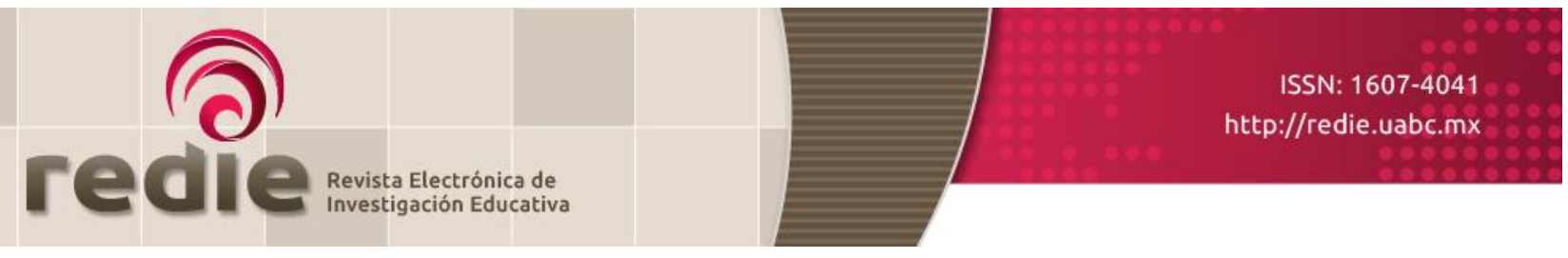

Vol. 20, Núm. 1, 2018

\title{
Instrumentos consistentes para la enseñanza de fracciones en 40. grado
}

\section{Consistent Instruments for Teaching Fractions in $4^{\text {th }}$ Grade}

Palmenia de la Cruz Rodríguez Rojas (1) prodriguez@userena.cl

Raimundo Olfos (2) raimundo.olfos@pucv.cl

(1) Universidad de La Serena

(2) Pontificia Universidad Católica de Valparaíso

(Recibido: 19 de abril de 2016; Aceptado para su publicación: 14 de noviembre de 2017)

Cómo citar: Rodríguez, P. de la C. y Olfos, R. (2018). Instrumentos consistentes para la enseñanza de fracciones en 40. grado. Revista Electrónica de Investigación Educativa, 20(1), 48-58. https://doi.org/10.24320/redie.2018.20.1.1358

\section{Resumen}

En Chile y, en general, para todos los sistemas educativos del mundo es conveniente disponer de instrumentos consistentes que midan los conocimientos que requiere un profesor para lograr que sus alumnos aprendan; instrumentos útiles para la formación inicial y continua del profesorado. El propósito de este estudio consistió en elaborar dos instrumentos consistentes, uno sobre el conocimiento profundo del profesor acerca de las fracciones y otro sobre el saber del profesor acerca del conocimiento que el alumno pone en juego al conceptualizar las fracciones en 40. grado. Los instrumentos fueron aplicados en dos oportunidades a grupos de 30 profesores de primaria y una vez a 20 estudiantes de la carrera de Pedagogía en Matemáticas. Tras los respectivos procedimientos psicométricos se obtuvieron dos instrumentos, de 12 y 10 preguntas, con una consistencia interna de 0.74 y 0.75 alfa de Cronbach.

Palabras clave: Fracciones, validación de instrumentos, enseñanza de las Matemáticas.

\section{Abstract}

In education systems both in Chile and worldwide, it is desirable to have consistent instruments to measure the knowledge required by teachers to help students learn. These would be useful instruments for teachers' initial and ongoing training. The purpose of this study was to develop two consistent instruments, one on teachers' in-depth knowledge of fractions, and one on teachers' understanding of the knowledge students bring into play when conceptualizing fractions in $4^{\text {th }}$ grade. The instruments were administered on two occasions to groups of 30 elementary school teachers, and once to 20 students from the pedagogy in mathematics degree. After completing the corresponding psychometric procedures, two instruments with 12 and 10 questions were obtained, with an internal consistency of Cronbach's alpha 0.74 and 0.75 . 


\section{Introducción}

En el informe de la Organización de las Naciones Unidas para la Educación, la Ciencia y la Cultura (UNESCO, 2012) se declara que los profesores son la clave para el desarrollo sostenible de la educación. Diversas investigaciones han puesto de manifiesto la relevancia de la calidad de los profesores respecto a los resultados de aprendizaje de los alumnos (Eurydice, 2013).

El estudio Teacher Education Study in Mathematics (TEDS-M) revela que Chile no está preparando a sus futuros profesores de educación primaria para enseñar matemáticas. Algunos factores que pueden explicar esos resultados son la insuficiencia de conocimientos matemáticos adquiridos en la enseñanza media (Ávalos y Matus, 2010).

En Chile, a los profesores noveles se les están haciendo evaluaciones masivas que miden conocimientos disciplinarios y didácticos, pero se requieren instrumentos consistentes para la evaluación del profesorado. Considerando los recientes hallazgos internacionales -que coinciden en identificar al profesor como el principal factor para el mejoramiento en la calidad de la educación (UNESCO, 2012; Bruns y Luque, 2014), y la necesidad de disponer de instrumentos de calidad que midan los conocimientos del profesorado- esta investigación tuvo por objetivo elaborar dos instrumentos consistentes sobre el conocimiento del profesor acerca de la enseñanza de las fracciones en 40 . grado.

Shulman (1986) es precursor en identificar el conocimiento que requiere un profesor para enseñar un contenido, al que denomina "conocimiento pedagógico del contenido" (CPC), reconociéndolo como "la forma particular del conocimiento que incorpora el aspecto del contenido que guarda más relación con la enseñanza". Así, desde que Shulman identificara el CPC, en las últimas tres décadas diversos investigadores han contribuido a precisar el dominio de los contenidos requeridos para enseñar matemáticas (Ball, 1990; Ball, 2000; Ball, Hill y Bass, 2005; Ball, Thames y Phelps, 2008; Carrillo, Climent, Contreras y Muñoz-Catalán, 2013; Hill y Ball, 2004; Rowland, Huckstep y Thwaites, 2005; Hill, Ball y Schilling, 2008; Krauss, 2007; Ma, 1999).

Este estudio se basó en dos subdominios de conocimientos del modelo Mathematical Knowledge for Teaching (MKT) (Ball et al., 2008): uno es el conocimiento especializado del contenido y otro es el conocimiento del contenido y de los estudiantes, específicamente se analizó el conocimiento matemático para la enseñanza.

Parece ser evidente que un profesor debe manejar los conocimientos que enseña; sin embargo, hay investigaciones que ponen de manifiesto la insuficiencia en temas elementales para la enseñanza de las matemáticas (Ball 1990; Ma, 2010; Sorto, Marshall, Luschei y Carnoy, 2009).

Mediante un estudio de corte cualitativo, Ma (1999) comparó el conocimiento que ponen en juego en la tarea de enseñar profesores estadounidenses y profesores chinos. Ma analiza el tipo de comprensión que distingue a los dos grupos de profesores: uno es procedimental, es decir, saber cómo realizar un algoritmo, y el otro es conceptual, es decir, saber por qué tiene sentido matemáticamente realizar ese algoritmo.

Según Ma (1999), corroborado por MT21 (2007), un factor clave para otorgar educación matemática de calidad es un conocimiento profundo del contenido matemático por parte del profesor. Para promover el aprendizaje de las matemáticas los profesores deben tener una comprensión profunda de las matemáticas fundamentales (CPMF). En este estudio adaptamos las características de CPMF de Ma (2010) y presentamos las categorías del conocimiento profundo de las fracciones (Cprofu-f):

- Conectividad. El profesor realiza conexiones explícitas entre los conceptos y los procedimientos matemáticos. Por ejemplo, propone ordenar fracciones en la recta numérica.

- Múltiples perspectivas. El profesor aprecia varios enfoques para una solución, y puede dar explicaciones matemáticas para esos enfoques. Los profesores muestran una comprensión 
flexible de las fracciones, por ejemplo, presentan diversas representaciones en contextos discretos y/o continuos.

- Ideas básicas. El profesor es consciente de los conceptos y principios matemáticos. Los profesores tienden a repasar y reforzar estas ideas básicas. Por ejemplo, plantea situaciones de fracciones en que la unidad difiere de 1.

- Coherencia longitudinal. El profesor muestra una comprensión fundamental del currículo escolar, da oportunidad de repasar conceptos previos y sentar las bases de un concepto que será aprendido en el futuro. Por ejemplo, comprende la importancia de la propiedad de densidad en los números reales.

En este estudio se aborda particularmente la enseñanza de las fracciones, tema recurrente en los niveles escolares primario a terciario, central en el desarrollo del pensamiento proporcional, y en el estudio de los números racionales, los porcentajes, los decimales y las razones (Cortina, Cardoso y Zúñiga, 2012).

Las fracciones son difíciles de aprender y de enseñar, su complejidad ha llevado a muchas investigaciones (Behr, Harel, Post y Lesh, 1993; Block y Solares, 2001; Carpenter, Fennema y Romberg, 1993; Fandiño, 2009; Freundenthal, 1983; Kieren, 1993; Rojas, Flores y Carrillo, 2011; Salazar, Martinic y Maz, 2011; Streefland, 1991; Valdemoros, 2010). Las fracciones se asocian a diversas situaciones y toman distintos significados, por ejemplo, parte-todo, cociente, medida, razón y operador, lo que las ubica en un contexto de aprendizaje complejo que demanda altas exigencias cognitivas para su conceptualización (Gallardo, González y Quispe, 2008).

El conocimiento que el profesor alcanza respecto a la relación de los alumnos con el contenido, es decir, errores, dificultades y estrategias utilizadas por los estudiantes, es uno de las componentes del conocimiento didáctico del profesor. Este conocimiento docente considera cómo los alumnos razonan y aprenden un contenido en particular (Artigue, Douady, Moreno y Gómez, 1995; Carrillo et al., 2013; Hill et al., 2008).

Con base en la investigación de Hill et al. (2008), en este estudio las características del saber del profesor acerca del conocimiento que el alumno pone en juego al conceptualizar las fracciones (Cconceptua-F) son las siguientes:

1) Conocimiento de errores comunes de los alumnos: El profesor identifica y obtiene explicaciones de los errores de los alumnos, advierte que los errores surgen del contenido.

2) Conocimiento de las dificultades de los alumnos: El profesor identifica y comprende cuáles son las dificultades o barreras más frecuentes de los alumnos y que inducen al error.

3) Conocimientos adquiridos por los alumnos: El profesor conoce las conceptualizaciones y los conocimientos previos adquiridos del alumno; por ejemplo, el profesor sabe lo que son capaces de hacer lo estudiantes de tercer grado.

4) Estrategias usadas por los alumnos: El profesor está familiarizado con las posibles soluciones que utilizarán sus alumnos al resolver una cuestión matemática.

\subsection{Marco conceptual para la construcción de los instrumentos}

En la presente investigación utilizamos nociones del modelo del conocimiento para la enseñanza de matemáticas propuesto por Hill et al. (2008), enriquecido con las ideas de Ma (1999). Las dimensiones del marco conceptual se declaran en los siguientes términos (ver figura 1); se operacionaliza el conocimiento profundo de las fracciones presentes en el currículo y para conceptualizar la dimensión Cprofu-F utilizaremos las características del conocimiento del profesor respecto a la conectividad, múltiples perspectivas, ideas básicas y coherencia longitudinal. Además, se operacionaliza el conocimiento del 
profesor acerca del saber del alumno Cconceptua-F, en cuanto los conocimientos adquiridos, a las dificultades, errores, estrategias usuales en el tema de las fracciones y el conocimiento del currículo.

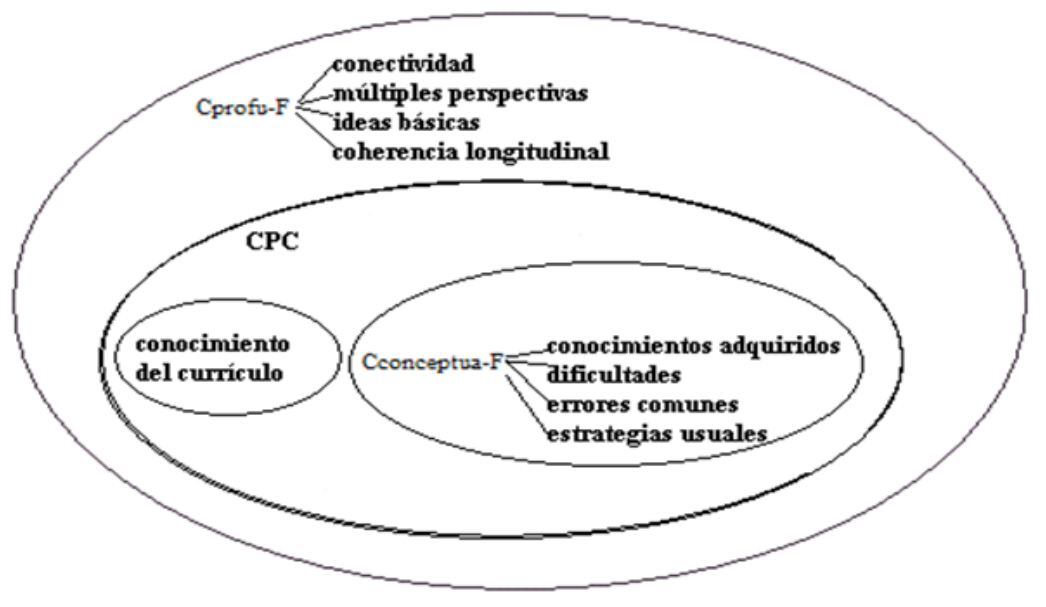

Figura 1. Esquema del marco conceptual.

\section{Método}

Este estudio se circunscribe al contenido de las fracciones según lo que establece el currículo chileno en el Programa de Estudio de 4o. grado (Mineduc, 2003). En la tabla I se observa que tales contenidos están presentes en la construcción de las preguntas de los instrumentos, proveyendo validez de contenido.

Tabla I. Contenidos mínimos obligatorios para fracciones 4o. grado*

\begin{tabular}{l|c|c}
\hline $\begin{array}{l}\text { Contenidos del Programa 4o. grado } \\
\text { Unidad fracciones }\end{array}$ & $\begin{array}{c}\text { Test Cprofu-F } \\
\text { Pregunta \# }\end{array}$ & $\begin{array}{c}\text { Test Cconceptua-F } \\
\text { Pregunta \# }\end{array}$ \\
\hline Situaciones de reparto equitativo & $11-12$ & $2-9$ \\
Lectura y escritura de fracciones & $4-5$ & $6-7$ \\
\hline Familias de fracciones de igual valor & $9-10$ & $3-8$ \\
Ubicación de fracciones mayores que la unidad en la recta numérica & 3 & 10 \\
\hline Uso de fracciones para precisar la descripción de la realidad & $6-7-8$ & 1 \\
\hline Total & 12 & 10 \\
\hline
\end{tabular}

*Fuente: Mineduc (2003).

El instrumento para medir el Cprofu-F contiene preguntas referidas a las cuatro categorías (ver tabla II). Lo que ofrece validez de contenido del instrumento en relación al constructo Cprofu-F. 
Tabla II. Categorías contenidas en el instrumento sobre el Cprofu-F de los profesores

\begin{tabular}{ll}
\hline \multicolumn{1}{c}{ Cprofu-F } & Pregunta \# \\
\hline Conectividad & $1-2-3$ \\
Múltiples perspectivas & $6-9-10$ \\
Ideas básicas & 4-5-11-12 \\
Coherencia longitudinal & $7-8$ \\
\hline
\end{tabular}

El instrumento para medir el Cconceptua-F contiene preguntas referidas a las cuatro categorías (ver tabla III), lo que ofrece validez de contenido al instrumento en relación al constructo Cconceptua-F.

Tabla III. Categorías Cconceptua-F contenidas en el instrumento sobre el Cconceptua-F de los profesores

\begin{tabular}{ll}
\hline \multicolumn{1}{c}{ Cconceptua-F } & No. de la pregunta \\
\hline Conocimientos Adquiridos & $4-5$ \\
Dificultades más frecuentes de los alumnos & $9-10$ \\
Errores posibles de los alumnos & $3-6-7-8$ \\
Estrategias usuales de los alumnos & $1-2$ \\
\hline
\end{tabular}

\subsection{Etapas y procedimientos}

Para analizar el grado de dificultad y discriminación de las preguntas se utilizaron los parámetros establecidos para la aceptación de preguntas, planteados por Guilford (1975) donde el rango aceptable para el índice de dificultad oscila entre 0.2 y 0.85 , y en el caso de la discriminación se asume un índice mínimo de 0.3. También consideramos a Backhoff, Larrazolo y Rosas (2000), quienes clasifican las preguntas según su nivel de dificultad, agrupándolas como "altamente difíciles" (<0.32); "medianamente difíciles" (0.33 a 0.52); "de dificultad media" (0.53 a 0.73); "medianamente fáciles" (0.74 a 0.86); y "altamente fáciles" (>0.86). Backhoff et al. (2000) señalan que entre más alto es el índice de discriminación, la pregunta diferenciará mejor a las personas con altas y bajas calificaciones.

1a. etapa: Elección de un instrumento desarrollado y probado. Se comenzó con un instrumento que constaba de 37 preguntas relativas al conocimiento matemático y didáctico sobre fracciones (Olfos, Guzmán y Galbiati, 2010). Del total de preguntas de ese instrumento se eligieron 23 preguntas pertinentes para la investigación, posteriormente se analizaron en función de sus índices de discriminación y de dificultad, y se mejoró su redacción.

2a. etapa: Revisión y redacción de preguntas Cprofu-F. De las 23 preguntas elegidas, se consideran 10 preguntas relativas al conocimiento del profesor acerca de las fracciones, 4 de las preguntas no fueron modificadas y 6 fueron adaptadas. Se agregaron 4 preguntas con el propósito de cubrir contenidos del currículo. Las preguntas se aparearon para medir lo mismo, esto permitió construir el instrumento Cprofu-F con 14 preguntas.

3a. etapa: Construcción y redacción de preguntas de Cconceptua-F. De las 23 preguntas seleccionadas, se eligieron 13 preguntas relativas al conocimiento del profesor respecto del saber del alumno, las cuales se agruparon de diversas formas para ver cuál de ellas presentaba mejor índice de discriminación y dificultad. Finalmente se eligieron 6 preguntas que fueron modificadas, luego se duplicaron las preguntas para medir lo mismo, se revisó la redacción y los distractores. Esto permitió construir el instrumento final Cconceptua-F constituido por 10 preguntas.

4a. Etapa: Primera aplicación del instrumento Cprofu-F y Cconceptua-F. El instrumento Cprofu-F fue respondido por 30 profesores de primaria y el instrumento Cconceptua-F fue respondido por 27 de los 30 profesores de primaria. 
5a. Etapa: Modificación del instrumento Cconceptua-F. La consistencia de Cprofu-F fue aceptable, no así la de Cconceptua-F, por lo que se examinaron y mejoraron los enunciados de las preguntas.

6a. Etapa: Segunda aplicación de los instrumentos Cprofu-Fy Cconceptua-F. Los instrumentos se aplicaron a 30 profesores de primaria y posteriormente a 20 estudiantes de la carrera de pedagogía en matemática

\section{Resultados}

Se obtuvo un instrumento Cprofu-F con una confiabilidad aceptable, estimada con el coeficiente alfa de Cronbach 0.74 y un instrumento Cconceptua-F con una confiabilidad también aceptable, estimada con el 0.75. En la tabla IV se muestran los índices de dificultad y discriminación de los instrumentos Cprofu-F y Cconceptua-F.

Tabla IV. Índices de discriminación y dificultad de los instrumentos Cprofu-F y Cconceptua-F

\begin{tabular}{cccccc}
\hline $\begin{array}{c}\text { Pregunta } \\
\text { Cprofu-F }\end{array}$ & $\begin{array}{c}\text { Índice de } \\
\text { dificultad }\end{array}$ & $\begin{array}{c}\text { Índice de } \\
\text { discriminación }\end{array}$ & $\begin{array}{c}\text { Pregunta } \\
\text { Cconceptua-F }\end{array}$ & $\begin{array}{c}\text { Índice de } \\
\text { dificultad }\end{array}$ & $\begin{array}{c}\text { Índice } \\
\text { discriminación }\end{array}$ \\
\hline 1 & 0.9 & 0.4 & 1 & 0.8 & 0.2 \\
2 & 0.7 & 0.3 & 2 & 0.6 & 0.4 \\
3 & 0.6 & 0.9 & 3 & 0.7 & 0.4 \\
4 & 0.7 & 0.6 & 4 & 0.4 & 0.6 \\
5 & 0.8 & 0.5 & 5 & 0.6 & 0.7 \\
6 & 0.6 & 0.8 & 6 & 0.7 & 0.7 \\
7 & 0.8 & 0.4 & 7 & 0.7 & 0.6 \\
8 & 0.8 & 0.3 & 8 & 0.5 & 0.8 \\
9 & 0.8 & 0.3 & 9 & 0.5 & 0.9 \\
10 & 0.8 & 0.4 & 10 & 0.7 & 0.8 \\
11 & 0.8 & 0.4 & & & \\
12 & 0.8 & 0.5 & & & \\
\hline
\end{tabular}

Con el fin de ilustrar el contenido de los instrumentos se presenta una pregunta de Cprofu-F y una pregunta de Cconceptua-F.

La pregunta 7 del instrumento Cprofu-F (ver tabla V) se relaciona con la categoría coherencia longitudinal, en la cual la respuesta del profesor debe reflejar que no sólo sabe lo que enseña en cierto nivel, sino que maneja un conocimiento de todo el currículo. El profesor que responde que muchas fracciones cumplen esa condición, sabe que $\mathrm{Q}$ es denso en $\mathrm{R}$.

Tabla V. Pregunta 7 Cprofu-F

\begin{tabular}{|l|}
\hline Juan busca una fracción entre $7 / 8$ y 1 , ¿qué frase es correcta? \\
a. No la encontrará, puesto que $8 / 8$ es igual a 1. \\
b. Sólo es posible encontrar una fracción entre esos \\
números. \\
c. Muchas fracciones cumplen esa condición. \\
d. Ninguna de las anteriores. \\
Respuesta correcta: c. \\
Categoría: Coherencia Longitudinal
\end{tabular}


La pregunta 10 del instrumento Cconceptua-F (ver tabla VI), corresponde a la categoría conocimiento del profesor sobre las dificultades frecuentes de los alumnos. La respuesta correcta del profesor se basa en su conocimiento para comprender la dificultad común que presentan los alumnos en este tipo de preguntas. La literatura advierte que los niños no siempre comprenden la representación de las fracciones en la recta numérica. El profesor que responde correctamente se da cuenta que el entero es dos, y que el alumno usualmente no ve dos enteros.

Tabla VI. Pregunta 10 Cconceptua-F

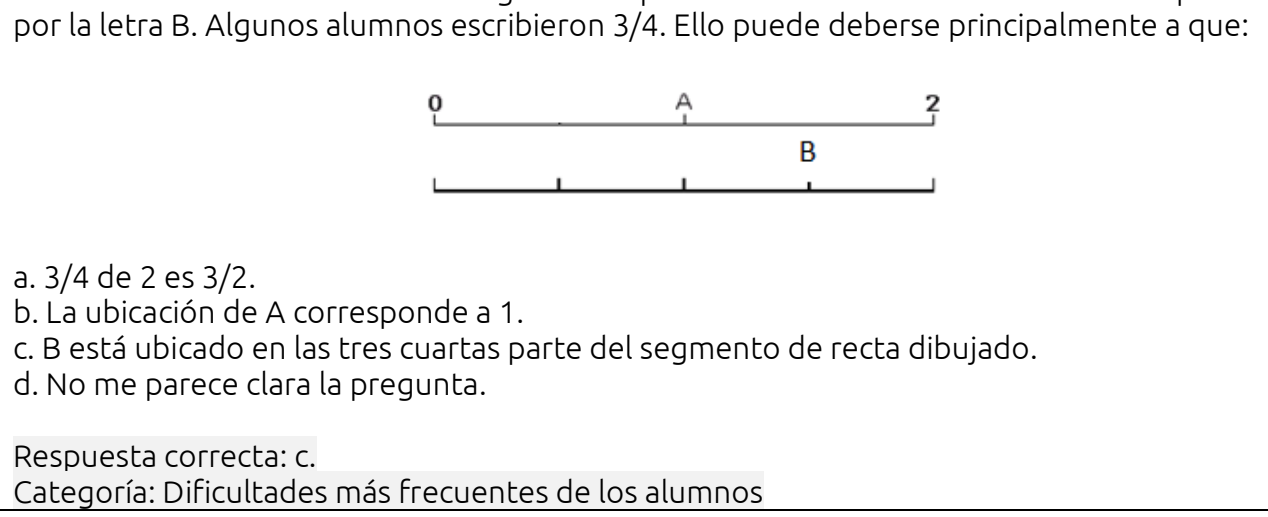
por la letra B. Algunos alumnos escribieron 3/4. Ello puede deberse principalmente a que:

a. $3 / 4$ de 2 es $3 / 2$.

b. La ubicación de A corresponde a 1.

c. B está ubicado en las tres cuartas parte del segmento de recta dibujado.

d. No me parece clara la pregunta.

Respuesta correcta: c

Categoría: Dificultades más frecuentes de los alumnos las distancias entre las marcas son iguales. La profesora solicitó escribir la fracción representada

\section{Discusión y conclusiones}

El estudio se enfocó en la construcción de dos instrumentos consistentes, uno que evalúa el conocimiento profundo del contenido acerca de las fracciones enmarcado en el currículo, Cprofu-F, y otro que evalúa el saber del profesor respecto al conocimiento que el alumno pone en juego al conceptualizar las fracciones en 40 . básico, Cconceptua-F.

El análisis de los índices de dificultad y discriminación, además de la revisión de los distractores y de la redacción de las preguntas del instrumento inicial (Olfos et al., 2010) permitieron dilucidar que las preguntas referentes al conocimiento del contenido que presentaban mejores índices de dificultad y discriminación medían un conocimiento matemático que difiere en ciertos aspectos al presentado en otras investigaciones relacionadas con el tema. Por ejemplo, Ball et al. (2008) utilizan la expresión "conocimiento matemático" para enseñar, distinguiendo las componentes, "conocimiento del contenido común" (cck) y "conocimiento del contenido específico" (sck). Si el profesor reconoce una respuesta incorrecta es el CCK, mientras que dimensionar la naturaleza del error es el sck, si el profesor se basa principalmente en su conocimiento matemático.

El instrumento inicial del proyecto FONIDE (Fondo de Investigación y Desarrollo en Educación) presentaba preguntas que medían las componentes del cck y del sck propuestos por Ball (2008), estas preguntas presentaban grados de dificultad y discriminación aceptable, pero además advertimos que aquellas preguntas que presentaban mejores índices de dificultad y de discriminación medían un conocimiento matemático del profesor que se aproximaba más a la idea de comprensión profunda de las matemáticas fundamentales de Ma (2010). Asumimos que este saber del profesor es un tipo "especial" de conocimiento matemático para la enseñanza, que denominamos "conocimiento profundo", y supusimos que este conocimiento se podía evaluar confiablemente. 
Al analizar las preguntas Cconceptua-F del instrumento inicial FONIDE, advertimos que las que mejor discriminaban eran aquellas que medían el conocimiento profundo referente a la comprensión conceptual del profesor, estos hallazgos nos condujeron a elaborar un esquema del marco conceptual en base a los objetivos presentados. En este estudio se presenta un esquema que difiere en parte del modelo matemático para la enseñanza de Hill et al. (2008), asumimos que el Cconceptua-F está contenido en el CPC, y este a su vez está contenido dentro del Cprofu-F, lo que es consistente con las ideas de Shulman (1986), que ubica el CPC entre el conocimiento del contenido y el conocimiento pedagógico. Hill et al. (2008) diferencian el conocimiento del contenido, del conocimiento pedagógico del contenido. El conocimiento de los estudiantes y el contenido (KCS), se encuentra dentro del CPC.

La construcción del esquema del marco conceptual, además de las investigaciones expuestas en este trabajo, permitió construir las preguntas de los instrumentos Cprofu-F y Cconceptua-F. El instrumento Cprofu-F mostró confiabilidad aceptable en las tres aplicaciones, por lo que no fue modificado. Este resultado se acerca a los resultados de Ball (2008), que logró medir con éxito el conocimiento matemático para enseñar.

El análisis de la confiabilidad de la primera versión del instrumento Cconceptua-F, no era aceptable (alfa de Cronbach 0.6), como ocurre en otros estudios que declaran que el Cconceptua-F es una componente que resulta difícil medir. Por ejemplo, Ball y su grupo de investigación no lograron las propiedades sicométricas aceptables para medir el conocimiento de los estudiantes y el contenido (alfa de Cronbach 0.5). Al reflexionar respecto a las preguntas que presentaban mejor grado de dificultad y discriminación, advertimos que además de medir Cconceptua-F estas medían conocimiento profundo, lo que incidió en la adopción del marco conceptual esquematizado en la figura 1, y consecuentemente, en la reformulación de las preguntas del Cconceptua-F.

En síntesis, se evalúa las dimensiones Cprofu-F y Cconceptua-F, conformando dos instrumentos con validez de contenido y confiabilidad aceptable. El trabajo de validación presenta definición conceptual para medir el constructo, mostramos tres tablas de especificaciones que proporcionan validez de contenido a ambos instrumentos. Cabe hacer notar que estos instrumentos tienen validez teórica y permiten identificar cuál es el nivel de conocimiento de un profesor. Se postula que este es el conocimiento que requiere para enseñar. Pero no hay evidencias de que este conocimiento sea una condición necesaria o suficiente para favorecer los aprendizajes de los estudiantes o al menos llevar adelante una instrucción de calidad. En este sentido, emerge el desafío de recabar evidencias al respecto, ámbito de investigación al que se abren los resultados de este estudio.

\section{Agradecimientos}

Proyecto FONDECYT REGULAR 1111009 Mineduc.

Proyecto DIULS INICIACIÓN PI1415 Universidad de La Serena.

\section{Referencias}

Artigue, M., Douady, R., Moreno, L., y Gómez, P. (1995). Ingeniería didáctica en educación matemática. Un esquema para la investigación y la innovación en la enseñanza y el aprendizaje de las matemáticas. México: Grupo Editorial Iberoamérica

Ávalos, B. y Matus, C. (2010). La formación inicial docente en Chile desde una óptica internacional. Informe Nacional del Estudio Internacional IEA-TEDS-M. Santiago: Mineduc.

Backhoff, E., Larrazolo, N. y Rosas, M. (2000). Nivel de dificultad y poder de discriminación del Examen de Habilidades y Conocimientos Básicos (EXHCOBA). Revista Electrónica de Investigación Educativa, 2(1). Recuperado de https://redie.uabc.mx/redie/article/view/15

Ball, D. (2000). Bridging Practices: Intertwining content and pedagogy in teaching and learning to teach. Journal of Teacher Education, 51(3), 241-247. 
Ball, D. (1990). The mathematical understandings that prospective teachers bring to teacher education. The Elementary School Journal, 90(4), 449-466.

Ball, D., Hill, H.C., y Bass H. (2005). Knowing mathematics for teaching. Who knows mathematics well enough to teach third grade, and how can we decide? American Educator, 29(1), 14-17.

Ball, D., Thames, M., y Phelps, G. (2008). Content knowledge for teaching: what makes it special? Journal of Teacher Education, 59(5), 389-407.

Barber, M. y Mourshed, M. (2007). Cómo hicieron los sistemas educativos con mejor desempeño del mundo para alcanzar sus objetivos. Buenos Aires: PREAL-McKinsey \& Company. Recuperado de http://www.oei.es/historico/noticias/spip.php?article3077

Behr, M., Harel, G., Post, T. y Lesh, R. (1993). Rational numbers: toward a semantic analysis-emphasis on the operator construct. En T. Carpenter, E. Fennema y T. Romberg (Eds.), Rational numbers: an integration of research (pp. 13-47). Nueva Jersey, Nu: Lawrence Erlbaum.

Block, D. y Solares, D. (2001). Las fracciones y la división en la escuela primaria: análisis didáctico de un vínculo. Educación Matemática, 13(2), 5-30.

Bruns, B. y Luque, J. (2014). Profesores excelentes: cómo mejorar el aprendizaje en América Latina y el Caribe. Serie del Foro sobre Desarrollo de América Latina. Washington: Banco Mundial.

Carpenter, T., Fennema, E. y Romberg, T. (Eds.). (1993). Rational numbers: an integration of research. Nueva Jersey, NJ: Lawrence Erlbaum.

Carrillo, J., Climent, N., Contreras, L., y Muñoz-Catalán, M. (2013). Determining specialized knowledge for mathematics teaching. En B. Ubuz, C. Haser y M. A. Mariotti (Eds.), Proceedings VIII Congress of the European Society for Research in Mathematics Education (CERME 8) (pp. 2985-2994). Turquía: Middle East Technical University.

Cortina, J., Cardoso, E. y Zúñiga, C. (2012). El significado cuantitativo que tienen las fracciones para estudiantes mexicanos de 6o. de primaria. Revista Electrónica de Investigación Educativa, 14(1), 70-85. Recuperado de https://redie.uabc.mx/redie/article/view/297/460

Eurydice (2013). Key data on teachers and school leaders. Eurydice Report. Luxemburgo: Publications Office European Union. Recuperado de http://eacea.ec.europa.eu/education/eurydice/documents/key data series/151EN.pdf

Fandiño, M. I. (2009). Las fracciones. Aspectos conceptuales y didácticos. Bogotá: Magisterio.

Freundenthal, H. (1983). Didactical phenomenology of mathematical structures. Dordrecht, Países Bajos: Reidel Publishing Company.

Gallardo, J., González, J. y Quispe, W. (2008). Interpretando la comprensión matemática en escenarios básicos de valoración. Un estudio sobre las interferencias en el uso de los significados de la fracción. Revista Latinoamericana de Investigación en Matemática Educativa, 11(3), 355-382.

Guilford, J. P. (1975). Psycometric methods (2a. Ed.). Bombay, Nueva Delhi. Tata McGraw-Hll.

Hill, H. y Ball, D. (2004). Learning mathematics for teaching: results from California's mathematics professional development institutes. Journal for Research in Mathematics Education, 35(5), 330- 351. 
Hill, H., Ball, D. y Schilling, S. (2008). Unpacking pedagogical content knowledge: conceptualizing and measuring teachers' topic-specific knowledge of students. Journal for Research in Mathematics Education, 39(4), 372-400.

Kieren, T. (1993). Rational and fractional numbers: from quotient fields to recursive understanding. En T. Carpenter, E. Fennema y T. Romberg (Eds.), Rational numbers: An integration of research (pp. 49-84). Nueva Jersey, NJ: Lawrence Erlbaum.

Krauss, S. (2007). Wie professionsspezifisch sind das fachdidaktische Wissen und das Fachwissen von Mathematiklehrkräften? Beiträge zum Mathematikunterricht bei der Gesellschaft für Didaktik der Mathematik. [¿Qué tan profesional es el conocimiento didáctico de las asignaturas y la experiencia de los profesores de matemáticas? Contribuciones a lecciones de matemáticas en la Sociedad de Didáctica de las Matemáticas]

Ma, L. (1999). Knowing and teaching elementary mathematics: Teachers' understanding of fundamental mathematics in China and the United States. Mahwah, NJ: Lawrence Erlbaum.

Ma, L. (2010). Conocimiento y enseñanza de las matemáticas elementales. La comprensión de las matemáticas fundamentales que tienen los profesores en China y los EE.UU. Academia Chilena de Ciencias.

Mineduc (2003). Programa de estudio 4o. Básico Matemática 2003. Chile: Autor. Recuperado de http://portales.mineduc.cl/index5 int.php?id portal=47\&id contenido=17116\&id seccion=3

MT21 (2007). Mathematic teaching in the 2st Century, The preparation gap: teacher education for middle school mathematics in six countries. Michigan University.

OCDE (2009). Política de educación y formación: los docentes son importantes. Atraer, formar y conservar a los docentes eficientes. París, Autor. Recuperado de http://www.waece.org/enciclopedia/2/Los\%20docentes\%20son\%20importantes.pdf

Olfos, R., Guzmán, I. y Galbiati, J. (2010). Conocimiento pedagógico del Contenido y su incidencia en la Enseñanza de la Matemática Nivel de Educación Básica (Informe Final Proyecto FONIDE Nº F410980). Chile: Mineduc.

Rojas, N., Flores, P., y Carrillo, J. (2011). Caracterización del conocimiento matemático de profesores de educación primaria y secundaria. En M. Marín y N. Climent (Eds.) Investigación en educación matemática. Comunicaciones de los grupos de investigación (pp. 395-400). XV Simposio de la SEIEM, Ciudad Real, España.

Rowland, T., Huckstep, P. y Thwaites, A. (2005). Elementary teachers' mathematics subject knowledge: The knowledge quartet and the case of Naomi. Journal of Mathematics Teacher Education, 8(3), 255-281.

Salazar, M. C., Martinic, S. y Maz, A. (2011). Avances de una investigación sobre los modelos, representaciones y recursos utilizados por profesores de primaria para las fracciones. En J. Lupiáñez, M. Cañadas, M. Molina, M. Palarea, y A. Maz (Eds.), Investigaciones en pensamiento numérico y algebraico e historia de la matemática y educación matemática (pp. 39-47). Universidad de Granada, España.

Shulman, L. S. (1986). Those who understand: Knowledge growth in teaching. Educational Researcher, 15(2), 4-14.

Sorto, M., Marshall, J., Luschei, T. y Carnoy, M. (2009). Teacher knowledge and teaching in Panama and Costa Rica: a comparative study in primary and secondary education. Revista Latinoamericana de Investigación en Matemática Educativa, 12(2), 251-290.

Streefland, L. (1991). Fractions in realistic mathematics education: a paradigm of developmental research. Países Bajos: Kluwer. 
UNESCO. (2012). Challenges in basic mathematics education. París: Autor. Recuperado de http://unesdoc.unesco.org/images/0019/001917/191776e.pdf

Valdemoros, M. (2010). Dificultades experimentadas por el maestro de primaria en la enseñanza de fracciones. Revista Latinoamericana de Investigación en Matemática Educativa, 13(4), 423-440. 